Discussion Paper No. 05-27

Optimal Abatement in Dynamic Multi-Pollutant Problems When Pollutants can be Complements or Substitutes

Ulf Moslener and Till Requate

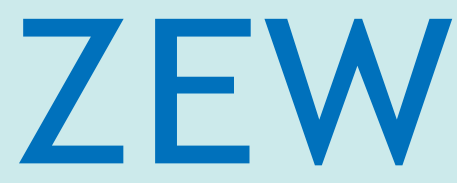

Zentrum für Europäische Wirtschaftsforschung $\mathrm{GmbH}$

Centre for European

Economic Research 
Discussion Paper No. 05-27

\title{
Optimal Abatement in Dynamic Multi-Pollutant Problems When Pollutants can be Complements or Substitutes
}

\author{
Ulf Moslener and Till Requate
}

Download this ZEW Discussion Paper from our ftp server:

ftp://ftp.zew.de/pub/zew-docs/dp/dp0527.pdf

Die Discussion Papers dienen einer möglichst schnellen Verbreitung von neueren Forschungsarbeiten des ZEW. Die Beiträge liegen in alleiniger Verantwortung der Autoren und stellen nicht notwendigerweise die Meinung des ZEW dar.

Discussion Papers are intended to make results of ZEW research promptly available to other economists in order to encourage discussion and suggestions for revisions. The authors are solely responsible for the contents which do not necessarily represent the opinion of the ZEW. 


\section{Non-Technical Summary}

Environmental problems are typically complex and involve not only one but many pollutants which usually have different characteristics. For such cases this analysis demonstrates that in general environmental policy based on the isolated analysis of only one pollutant may be qualitatively misleading.

We set up a partial model for several accumulating pollutants which cause different externalities but where abatement of different pollutants does not happen independently. In this regard the pollutants may be considered as complements or substitutes. For technologies which jointly abate different pollutants these may be called complements whereas for technologies which reduce emissions of one pollutant at the expense of higher emissions of another pollutant they can be called substitutes. Resulting optimal abatement paths are studied and illustrated for the case of two pollutants. These paths are found to be qualitatively different for complements and for substitutes.

Finally, we propose a simple policy rule that could be applied to implement the comparatively complex optimal emission paths. This policy rule uses the dynamic characteristics of the pollutants and combines them with a simple set of time invariant rules. 


\title{
OPTIMAL ABATEMENT IN DYNAMIC MULTI-POLLUTANT PROBLEMS WHEN POLLUTANTS CAN BE COMPLEMENTS OR SUBSTITUTES *
}

\author{
ULF MOSLENER ${ }^{\dagger}$ AND TILL REQUATE ${ }^{\ddagger}$
}

APRIL 2005

\begin{abstract}
We analyze a dynamic multi-pollutant problem where abatement costs of several pollutants are not separable. The pollutants can be either technological substitutes or complements. Environmental damage is induced by the stock of accumulated pollution. We find that optimal emission paths are qualitatively different for substitutes and complements. We derive general properties governing optimal emission paths and present numerical examples to illustrate our main results. In particular we find that optimal emission paths need not be monotonic, even for highly symmetric pollutants. Finally, we describe a comparatively simple method to implement the optimal path without explicitly knowing its shape.
\end{abstract}

JEL Classification: Q2, L5

Keywords: Multi-pollution, abatement technology, accumulating pollutants

${ }^{*}$ We are especially grateful to Harald Tauchmann for discussions and hints. The HeidelbergMannheim Graduate Programme acknowledges the support by the Deutsche Forschungsgemeinschaft $D F G$.

${ }^{\dagger}$ Centre for European Economic Research (ZEW), L7,1, 68161 Mannheim, phone (+49)-621-1235209, moslener@zew.de.

${ }^{\ddagger}$ Department of Economics, University of Kiel, Olshausenstr. 40, 24098 Kiel, phone (+49)-431-8804424, requate@bwl.uni-kiel.de. 


\section{INTRODUCTION}

The environmental policy literature usually concentrates on regulation of a single pollutant or assumes that different pollutants can be treated separately. Many production processes, however, are not only accompanied by the emissions of more than one pollutant, but modern abatement technologies also allow for joint abatement of several pollutants. An example is the catalytic converter for cars which jointly reduces $\mathrm{SO}_{2}, \mathrm{CO}$, and $\mathrm{NO}_{X}$. In this case we refer to pollutants which can be abated jointly as complements.

Other technologies reduce certain pollutants at the cost of increasing the output of at least one other pollutant. As an example particulate matter and $\mathrm{SO}_{2}$ emitted from power plants are abated by using considerable amounts of energy which in turn leads to an increase of $\mathrm{CO}_{2}$-emissions. Thus $\mathrm{SO}_{2}$ - and $\mathrm{CO}_{2}$-emissions can be considered as substitutes. A further example of substitutable pollutants is waste treatment where combustion, on the one hand, and deposition of waste, on the other, cause either carbon dioxide or methane emissions, respectively.

Emissions can also be considered as complements or substitutes if the inputs that cause these emissions in the production process are complements or substitutes in the usual sense. Since the choice of the input mix has to be done simultaneously, it is clear that also abatement decisions can in general not be made separately for each pollutant.

This paper studies the dynamic properties of optimal joint abatement paths and the corresponding policy rules when pollutants accumulate and when the social damage is caused by the several stocks of pollution. We show that, even if pollutants are symmetric with respect to both, their abatement costs and the damage they cause, optimal abatement paths will look different for substitutes and complements. Besides presenting some instructive numerical examples of optimal abatement paths for complements and substitutes, we derive general properties of the optimal emission/abatement paths. In particular we show for the case of two pollutants that optimal steady state emissions of the less harmful pollutant rise with the degree of substitutability while this effect is ambiguous for the more harmful pollutant. We also show that the optimal paths need not be monotonic, a property which does not emerge in the case of a single accumulating pollutant.

Finally, we discuss policy rules which determine how to deal with several interacting pollutants. We suggest to regulate a multi-pollutant problem by issuing tradeable permits since those can be implemented in a relatively simple way. For $n$ pollutants, the set of all optimal abatement paths - each path being generated by an $n$-vector of initial pollution stocks - can be considered as an $n$-dimensional manifold in a $2 n$ dimensional space. Instead of calculating this rather complex optimal path, it suffices 
for the regulator to have at hand a table of numbers representing a hyperplane which contains the optimal emission path. In the case of two pollutants, the table tells the regulator how to pick the optimal pair of permit quantities given any pair of pollution stocks. If the permit markets are competitive, market prices for permits reveal the optimal marginal social cost of pollution. In principle, a corresponding table can also be created if the regulator prefers to charge emission taxes.

To date, only few authors have treated multi-pollution problems. In a static framework Cansier and Cansier (1999), Endres (1985) and Repetto (1987) examine the case of several pollutants by employing the concept of iso-cost and iso-damage curves. In a dynamic context, multiple pollutant aspects have been studied above all for the greenhouse problem. In his world model, Nordhaus (2000) accounts for different greenhouse gases (GHGs), however, he limits the optimal choice of emissions to carbon dioxide $\left(\mathrm{CO}_{2}\right)$. Michaelis (1996) minimizes additively separable abatement cost of several GHGs under a given $\mathrm{CO}_{2}$ budget. Manne and Richels (2000) combine different model components, among those both economic and climate submodels, in order to form an intertemporal general equilibrium model to assess multi-gas- and $\mathrm{CO}_{2}$-only scenarios. They find that a multi-gas strategy benefits all developed regions except the economies in transition. Reilly et al. (1999) study multi-gas abatement strategies in a framework of an integrated global systems model. Furthermore, Böhringer et al. (forthcoming) investigate the efficiency gains from "what-flexibility" with respect to the emissions of carbon dioxide and methane within the frame of an integrated computable general equilibrium assessment. Burniaux (2000) focuses on the empirical determination of abatement cost by estimating the costs to reach the Kyoto targets under different flexibility regimes. He finds that restricting attention to $\mathrm{CO}_{2}$ only introduces an upward bias of cost estimations. Moslener and Requate (2001) characterize first best optimal emission paths if multiple accumulating pollutants interact with respect to social damage. By contrast to this paper they assume abatement costs to be additively separable. Different dynamic and physical properties of the pollutants are considered and found to influence both the behavior of optimal emissions and their shadow prices over time.

The paper is organized as follows. In the next section we outline the model. Section 3 characterizes optimal emission/abatement paths and possible policy rules followed by numerical simulations of optimal paths. Section 4 briefly discusses decentralized policy. In Section 5 we present our conclusions.

\section{The MOdeL}

We consider a model where economic activity causes several pollutants. We denote by $E_{i}$ the flow of emissions and by $S_{i}$ the accumulated stock of pollutant $i$, respectively. 
We assume that the damage depends on the stocks of the pollutants, whereas the abatement costs are a function of the flow of emissions. The pollutants accumulate according to the following equations of motion

$$
\dot{S}_{i}=E_{i}-\beta_{i} S_{i}
$$

where $0<\beta_{i}<1$ is the (constant) decay rate of pollutant $i=1, \ldots, n$.

We rule out interaction on the damage side by assuming that the environmental damage function is additively separable, i.e. $D\left(S_{1}, \ldots, S_{n}\right)=\sum D_{i}\left(S_{i}\right)$. In other words, the damage caused by an additional ton of pollutant $i$ is not influenced by any other pollutant.

By contrast, the abatement $\operatorname{costs} C\left(E_{1}, \ldots, E_{n}\right)$ depend on the whole vector of emissions. Restricting to the case of two pollutants we assume $C_{i}:=\frac{\partial C}{\partial E_{i}}<0$, where $E_{j} \leq E_{j}^{\max }$ for $j=1,2$, and $C_{i i}:=\frac{\partial^{2} C}{\left(\partial E_{i}\right)^{2}}>0$. Moreover, $C$ is twice continuously differentiable and strictly convex, implying $C_{11} C_{22}-\left[C_{12}\right]^{2}>0$. The sign of $C_{12}\left(=C_{21}\right)$ may go either way in general. The two pollutants are called substitutes if $C_{12}>0$ and complements if $C_{12}<0$, respectively. ${ }^{1}$

The social planner minimizes the discounted sum (the integral) of social cost arising over time:

$$
\min _{E_{1}(t), E_{2}(t)} \int_{0}^{\infty}\left[C\left(E_{1}, E_{2}\right)+D_{1}\left(S_{1}\right)+D_{2}\left(E_{2}\right)\right] e^{-r t} d t
$$

subject to the equation of motion of the accumulating stocks (2.1) for $i=1$, 2, where $r$ as usual is the social discount rate. The current value Hamiltonian is then given by

$$
H=C\left(E_{1}, E_{2}\right)+D_{1}\left(S_{1}\right)+D_{2}\left(S_{2}\right)+\lambda_{1}\left(E_{1}-\beta_{1} S_{1}\right)+\lambda_{2}\left(E_{2}-\beta_{2} S_{2}\right)
$$

where $\lambda_{i}$ are the costate variables. From the first-order conditions

$$
\begin{aligned}
\frac{\partial H}{\partial E_{i}} & =C_{i}^{\prime}\left(E_{i}, E_{j}\right)+\lambda_{i}=0 \\
-\frac{\partial H}{\partial S_{i}} & =\dot{\lambda}_{i}-\lambda_{i} r
\end{aligned}
$$

\footnotetext{
${ }^{1}$ This notion can be motivated as follows. Let us denote by $\lambda_{i}$ is the shadow price of abatement of pollutant $i$ under any kind of regulation. Then, given $E_{j}$, the firm sets $-C_{i}\left(E_{i}, E_{j}\right)=\lambda_{i}(*)$. If $E_{j}$ is relaxed, we see by implicitly differentiating $\left(^{*}\right)$ that $\partial E_{i} / \partial E_{j}=-C_{i j} / C_{i i}$ is positive for $C_{i j}<0$ (complements) and negative for $C_{i j}>0$ (substitutes) as intuition would suggest.
} 
and by eliminating the time derivative of the costate variable $(\dot{\lambda})$ the maximum principle leads to the corresponding Ramsey conditions. They read

$$
\begin{aligned}
& \dot{E}_{1}=\frac{C_{22}\left(D_{1}^{\prime}+C_{1}\left(\beta_{1}+r\right)\right)-C_{12}\left(D_{2}^{\prime}+C_{2}\left(\beta_{2}+r\right)\right)}{C_{11} C_{22}-\left[C_{12}\right]^{2}}, \\
& \dot{E}_{2}=\frac{C_{11}\left(D_{2}^{\prime}+C_{2}\left(\beta_{2}+r\right)\right)-C_{21}\left(D_{1}^{\prime}+C_{1}\left(\beta_{1}+r\right)\right)}{C_{11} C_{22}-\left[C_{12}\right]^{2}} .
\end{aligned}
$$

Together with the dynamic constraints (2.1) these Ramsey conditions (2.6) form a system of four differential equations. To calculate the optimal paths explicitly, we additionally need the initial conditions (i.e., initial stocks) and the transversality conditions (i.e., $\lim _{T \rightarrow \infty} \lambda_{i}(T)=0$ ) to rule out diverging paths. Note, that for additively separable costs, i.e. $C_{i j}=0$ for $i \neq j$, we obtain the case of two separate single pollutants, and (2.6) turns into the standard textbook case.

The goal of this analysis is to highlight the different behavior of the optimal emission paths and of the shadow prices of pollution (which correspond to the optimal emission taxes in a first-best scenario) for the cases where emissions are either complements or substitutes. For our purposes it suffices to take an easy specification for both the damage and the cost functions which allows us to characterize pollutants as complements or substitutes but which keeps the system linear. Hence, we assume a quadratic damage function, such that

$$
D_{i}\left(S_{i}\right)=\left(\alpha_{i} S_{i}\right)^{2}
$$

for $i=1,2$ where $\alpha_{i}$ indicates the harmfulness of pollutant $=i$. Abatement costs are taken bi-quadratic:

$$
C\left(E_{1}, E_{2}\right)=\frac{\eta_{1}}{2}\left(\bar{E}_{1}-E_{1}\right)^{2}+\frac{\eta_{2}}{2}\left(\bar{E}_{2}-E_{2}\right)^{2}+\omega\left(\bar{E}_{1}-E_{1}\right)\left(\bar{E}_{2}-E_{2}\right)
$$

for $E_{i}<E_{i}^{\max }$. Here $\bar{E}_{i}$ present the laisser-faire emission levels of the two pollutants. ${ }^{2}$ The parameters $\eta_{i}$ describe the marginal abatement cost (disregarding the other pollutant). The parameter $\omega$ is crucial and determines whether the pollutants are substitutes $(\omega>0)$ or complements $(\omega<0)$. To assure $\frac{\partial C}{\partial E_{i}}<0$ we assume $|\omega|<\eta_{i}$ for $i=1,2$.

Note, that w.l.o.g. the marginal abatement costs for pollutant 1 are linear in $E_{1}$ but shifted upwards or downwards by a higher level of $E_{2}$. The direction is determined by the sign of $\omega$ (upwards for substitutes, downwards for complements). The size of the shift is determined by the absolute value of $\omega$ and by the current emission level $E_{2}$ of the other pollutant.

For our purposes it would be ideal to vary the substitutability in the cost function without changing the marginal abatement costs. But since substitutability relates to

\footnotetext{
${ }^{2}$ For the usual assumptions $\left(C^{\prime}<0, C^{\prime \prime}>0\right)$ it is sufficient to set $E_{1}^{\max }=\bar{E}_{1}-\left|\frac{\omega}{\eta_{1}}\right| \bar{E}_{2}$ and $E_{2}^{\max }=\bar{E}_{2}-\left|\frac{\omega}{\eta_{2}}\right| \bar{E}_{1}$.
} 
a curvature property it cannot be altered without changing the first derivative as well. A variation of the substitutability in our model will therefore always induce two effects on the emissions $E_{i}$ :

(1) A level-effect, changing the overall emissions level due to the overall change in marginal abatement costs.

(2) A substitution-effect,changing the allocation of emissions between the two pollutants.

Accordingly, changing $\omega$ will shift the steady state in a twofold way.

\section{Optimal Policy for Compliments and Substitutes}

Since the system of differential equations to $b$ analised is linear, the general solution can be written in terms of the steady state $\left(E_{1}^{*}, E_{2}^{*}, S_{1}^{*}, S_{2}^{*}\right)$ and a linear combination of the four eigenvectors $\left(\vec{v}_{a}, \ldots, \vec{v}_{d}\right)$ :

$$
\left(\begin{array}{c}
E_{1}^{*}(t) \\
E_{2}^{*}(t) \\
S_{1}^{*}(t) \\
S_{2}^{*}(t)
\end{array}\right)=\left(\begin{array}{c}
E_{1}^{*} \\
E_{2}^{*} \\
S_{1}^{*} \\
S_{2}^{*}
\end{array}\right)+c_{a} \vec{v}_{a} e^{\lambda_{a} t}+c_{b} \vec{v}_{b} e^{\lambda_{b} t}+c_{c} \vec{v}_{c} e^{\lambda_{c} t}+c_{d} \vec{v}_{d} e^{\lambda_{d} t}
$$

Here $\lambda_{a}, \ldots, \lambda_{d}$ are the eingenvalues corresponding to the the eigenvectors $\vec{v}_{a}, \ldots, \vec{v}_{d}$ with the same index. The coefficients $c_{a}, \ldots, c_{d}$ have to be determined by using the transversality condition, i.e. restricting to solutions which satisfy the initial conditions and converge towards the steady state. As it is usual for these differential equations (and as we will see later from equation (5.15) in Appendix A), two of the eigenvalues have a negative sign and two have a positive sign. W.l.o.g., let $\lambda_{c}>0$ and $\lambda_{d}>0$. Hence, the corresponding coefficients $\left(c_{c}, c_{d}\right)$ have to be zero, otherwise these terms in equation (3.1) would diverge. Optimal motion towards the steady state can therefore be represented $\mathrm{as}^{3}$ :

$$
\left(\begin{array}{c}
E_{1}^{*}(t) \\
E_{2}^{*}(t) \\
S_{1}^{*}(t) \\
S_{2}^{*}(t)
\end{array}\right)=\left(\begin{array}{c}
E_{1}^{*} \\
E_{2}^{*} \\
S_{1}^{*} \\
S_{2}^{*}
\end{array}\right)+c_{a} \vec{v}_{a} e^{\lambda_{a} t}+c_{b} \vec{v}_{b} e^{\lambda_{b} t}
$$

where $\lambda_{a, b}<0$. The two coefficients $c_{a, b}$ have to be chosen such that the equation matches the initial pollutant stocks at $t=0$.

One can furthermore see from (3.2) that all optimal paths are located in a plane which is spanned by the two eigenvectors $\overrightarrow{v_{a}}$ and $\overrightarrow{v_{b}}$. This form also shows that it is possible to

\footnotetext{
${ }^{3}$ For further details on the role of eigenvalues and eigenvectors in optimal control see Chiang (1992) or Feichtinger and Hartl (1986).
} 
represent the optimal paths as phase-space trajectories moving simultaneously along $\vec{v}_{a}$ and $\vec{v}_{b}$ with speeds $\lambda_{a}$ and $\lambda_{b}$, respectively.

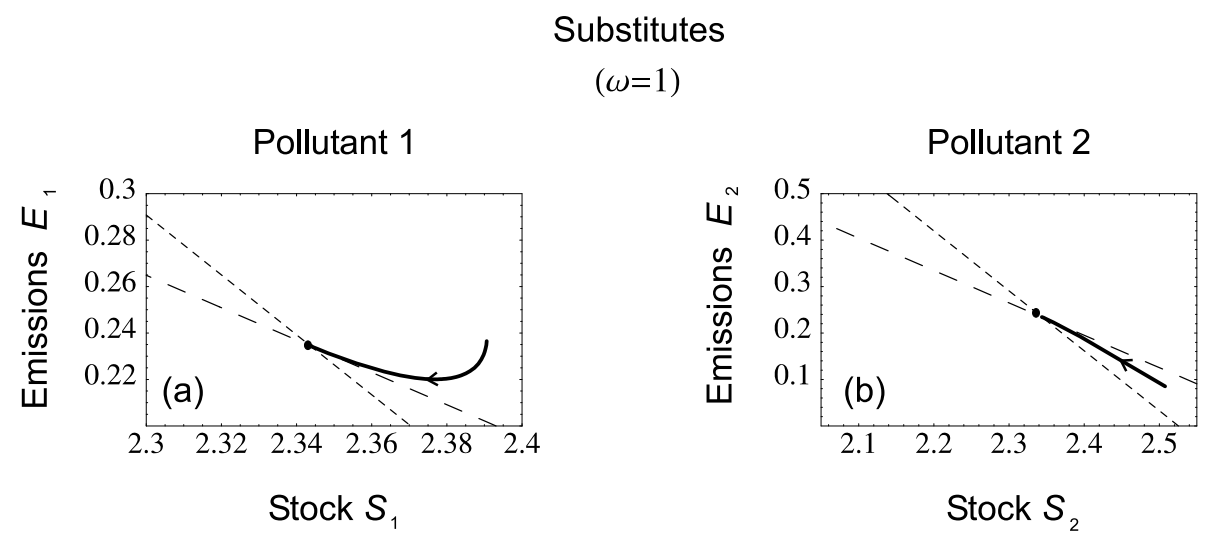

Figure 1. Substitutes $(\omega=1)$ : Optimal paths for a numerical example $(\eta=2 ; \beta=0.1 ; \alpha=1 ; r=0.06)$ in $E_{i}-S_{i}$-phase diagrams. Initial stocks are chosen to be $2 \%\left(S_{1}\right)$ and $7 \%\left(S_{2}\right)$, respectively, above the steady state.

3.1. Simulation Results for Complements and Substitutes. Before deriving some general properties of the optimal emission paths, we present some numerical simulations. Since the system is autonomous, we can display the properties of the optimal paths by a phase space diagram. For each numerical simulation in our analysis we will have to choose specific parameter values. Therefore, it is helpful to first consider the following special case:

Example 3.1. (1) The cost function is symmetric, i.e.

$$
\eta_{1}=\eta_{2}=\eta \text { and } \bar{E}_{1}=\bar{E}_{2}=\bar{E} .
$$

(2) The two pollutants have identical decay rates, i.e.

$$
\beta_{1}=\beta_{2}=\beta .
$$

(3) The two pollutants are equally harmful, i.e. the damage functions are identical

$$
\alpha_{1}=\alpha_{2}=\alpha .
$$

Note, that these three conditions define a two-pollutant-problem where the pollutants are identical, but distinguishable. We are free yet to choose $\omega$, which defines whether the pollutants are complements or substitutes. 
For all our numerical simulations we will assume that (3.3) - (3.5) hold and we will set $\eta=2, \beta=0.1, \alpha=1$, and $r=0.06$. Figure (1) illustrates the optimal paths of the example for the case of substitutes $(\omega=1)$ whereas figure (2) displays those for complements $(\omega=-1)$. In both scenarios initial stocks are chosen approximately two percent above the steady state level $S_{1}^{*}$ for pollutant 1, and seven percent above the steady state level $S_{2}^{*}$ for pollutant 2 . The bold lines with an arrow show the phase-space trajectories towards the steady state. The dashed lines mark the (projections of the) eigenvectors where the long-dashed line corresponds to the small (slow) stable eigenvector while the short-dashed line corresponds to the larger (fast) stable eigenvector. Correspondingly, the trajectories show that in the neighborhood of the steady state the fast movement is basically completed and the trajectory is governed by the direction of the slow eigenvector. Obviously there are striking qualitative differences between optimal emissions over time in the two cases displayed in the Figures 1 and 2. These differences must be driven by the complementarity and substitutability properties, respectively, since the two pollutants are identical in all other aspects.

\section{Complements}

$(\omega=-1)$

Pollutant 1

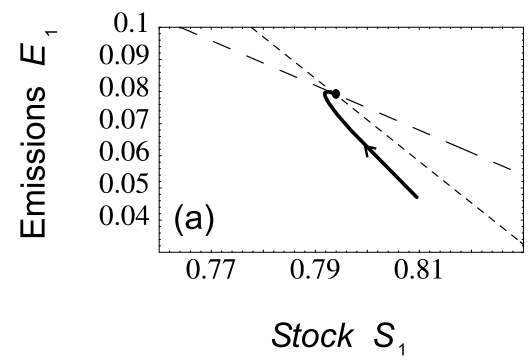

Pollutant 2

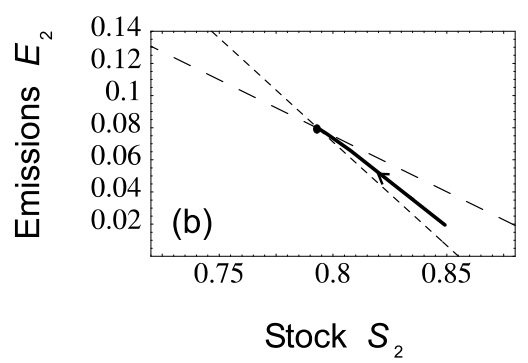

Figure 2. Complements $(\omega=-1)$ : Optimal paths for a numerical example $(\eta=2 ; \beta=0.1 ; \alpha=1 ; r=0.06)$ in $E_{i}-S_{i}$-phase diagrams. Initial stocks are chosen to be $2 \%\left(S_{1}\right)$ and $7 \%\left(S_{2}\right)$, respectively, above the steady state.

For substitutes (figure 1) we see that initially there will be a higher level of abatement for the pollutant for which the original stock is higher $\left(S_{2}\right)$ than for the other pollutant. As can be seen from the diagram for pollutant 1, this is done at the expense of initially higher emissions $E_{1}$ of pollutant 1 . This reflects the substitutability. As the larger stock $S_{2}$ shrinks, abatement of this pollutant will slightly be relaxed while abatement of the 
other pollutant will be increased. Eventually emissions of both pollutants converge from below toward the steady state on very similar paths.

For complements (figure 2) we observe a different behavior: The emission paths of both pollutants start with relatively sharp abatement which will then be relaxed fairly quickly. In the long run the emissions $E_{1}$ converge towards the steady state from above while $E_{2}$ converges from below.

Note, that although in both cases the initial stocks of pollutant 1 and pollutant 2 are chosen by $2 \%$ and $7 \%$ above the steady state levels, respectively, emissions $E_{1}$ of pollutant 1 undershoot the steady state level for substitutes and overshoot that level for complements.

(a)

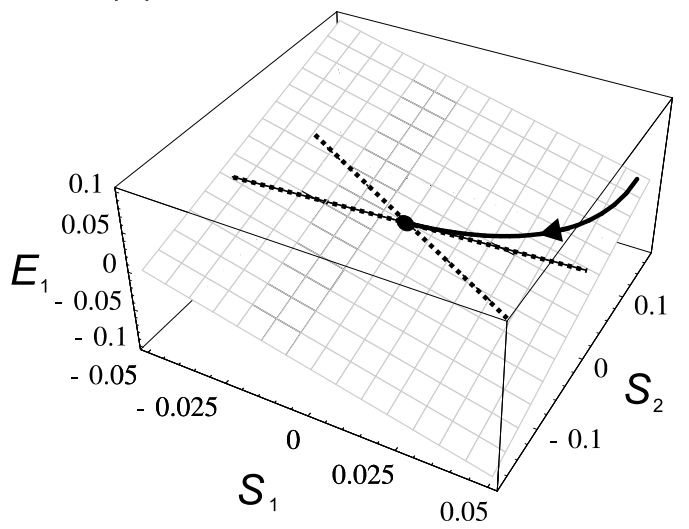

(b) Emissions $E_{2}$

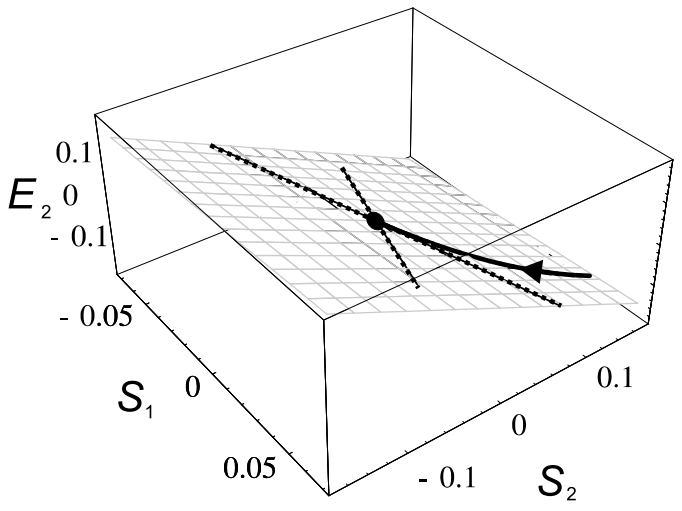

Figure 3. Optimal path, eigenvectors and optimal policy plane for substitutes $(\omega=1)$. The scenarios are identical to those shown in the previous figures. The axes $E_{i}, S_{1}, S_{2}$ indicate the deviation from the steady state.

Since the two-dimensional phase diagrams hide the impact of the second pollutant, it is useful to draw three-dimensional diagrams, plotting emissions against both stocks. We know from equation (3.1) that all optimal paths are located in a plane which is spanned by the eigenvectors $\vec{v}_{a}$ and $\vec{v}_{b}$. This plane - showing optimal emissions $E_{i}$ will be called the optimal policy plane for pollutant $i$. For each pair of stocks $S_{1}$ and $S_{2}$ the planes in (a) and (b) show the optimal emissions $E_{1}$ and $E_{2}$, respectively. It is important to notice that this does not only hold for the initial stocks but for the whole optimal path: For a given pair of initial stocks the optimal path is fully described by (i) the planes (in (a), (b) of figures 3 and 4) and (ii) by the dynamic constraints (for the motion on the planes). Note, that not every path one can draw on a policy plane is physically feasible, i.e. compatible with the dynamic constraints, but every physically 
feasible path that lies on the plane is optimal. This will be helpful later on for the implementation of the optimal path.

(a) Emissions $E_{1}$

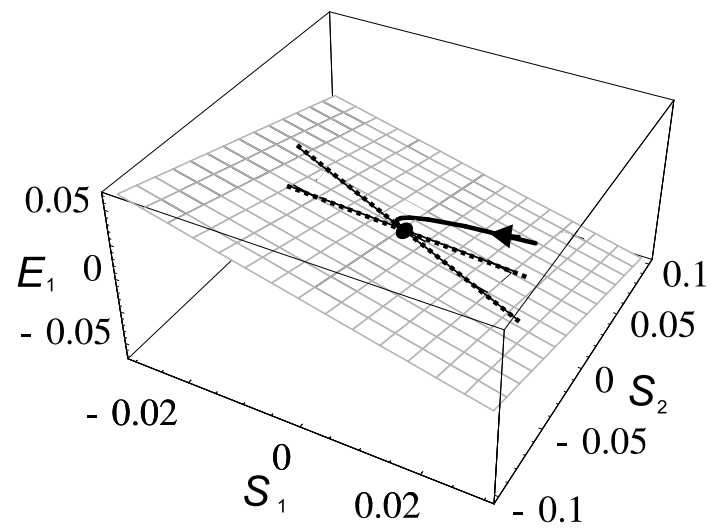

(b) Emissions $E_{2}$

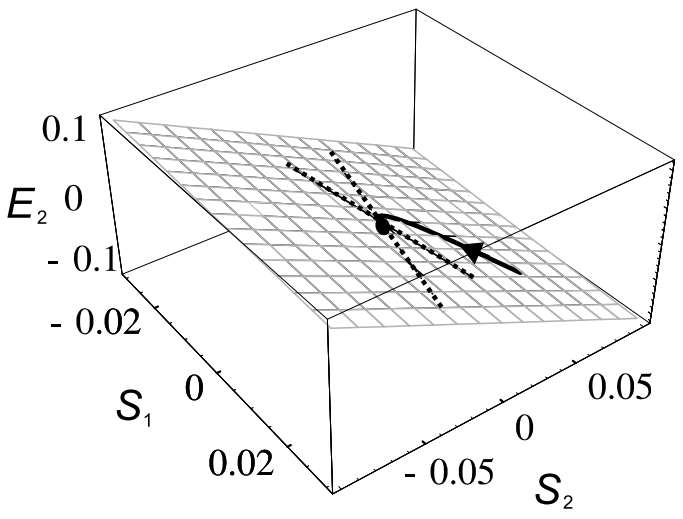

Figure 4. Optimal path, eigenvectors and optimal policy plane for complements $(\omega=-1)$. The scenarios are identical to those shown in the previous figures. The axes $E_{i}, S_{1}, S_{2}$ indicate the deviation from the steady state.

Figures 3 (substitutes) and 4 (complements) display our scenarios from figures 1 and 2 in such a way. The axes do not explicitly show the values of $E_{i}$ and $S_{i}$ but rather their deviation from the steady state $\left(E_{i}-E_{i}^{*}, S_{i}-S_{i}^{*}\right)$. In addition to the steady state (black dot), the optimal path (black line with arrow) and the eigenvectors (black dotted straight lines), which we know already from the previous figures 1 and 2, figures 3 and 4 also display the planes spanned by the eigenvectors $\vec{v}_{a}$ and $\vec{v}_{b}$ of equation (3.2). As we know, this is the plane that contains all the optimal paths (including the one that is drawn). Each optimal path is generated by a particular pair of initial conditions $\left(S_{1}(0), S_{2}(0)\right)$. The three-dimensional plots also display the the optimal paths from our previous example. ${ }^{4}$

\subsection{Qualitative differences of optimal paths for complements and substi-} tutes. To derive some qualitative differences between optimal paths for complements and substitutes we now abstract from the specific paths and directly examine the policy planes and eigenvectors instead. In figure 5 we display the planes for both complements (grey) and substitutes (white) in one diagram for the emissions of pollutant 1 (figure

\footnotetext{
${ }^{4}$ Note, that, e.g., figure 1 (a) is a projection of figure 3 (a) into the $E_{1}-S_{1}$ plane.
} 
5 (a)) and pollutant 2 (figure $5(\mathrm{~b})) .{ }^{5}$ These diagrams illustrate the difference between optimal policy for complements and substitutes. The planes are tilted in different directions intersecting along one steady state stock.

(a)

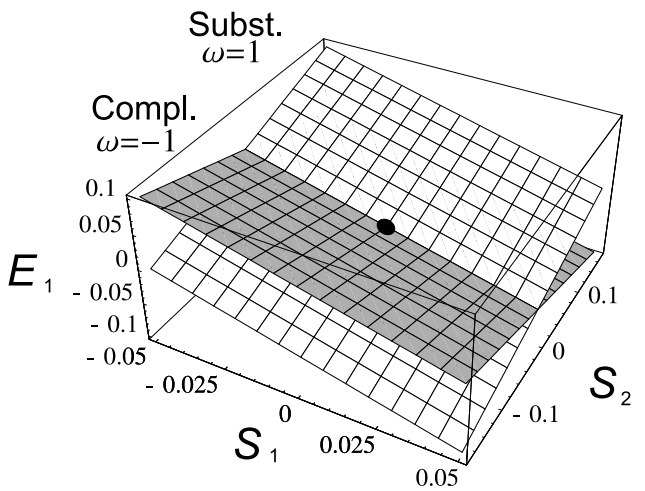

(b) Emissions $E_{2}$

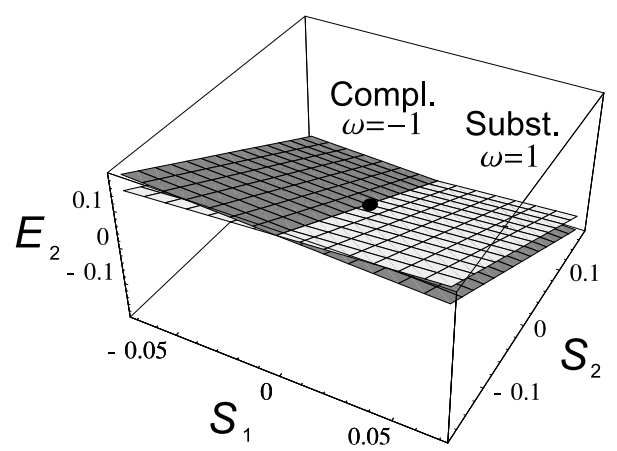

FiguRE 5. Three-dimensional policy rules for pollutants which are substitutes $(\omega=1)$ or complements $(\omega=-1)$ shown in one diagram for each pollutant.

We are now ready to derive some general properties of the actual optimal paths by analyzing the planes and eigenvectors for complements and substitutes. In figure 5 we notice that the intersection line of the optimal emission planes for complements and substitutes in the emissions $E_{1}$-diagram is located at $S_{2}=0$. The two planes are mirror-images of each other. Correspondingly, the intersection line in the emissions $E_{2}$-diagram is located at $S_{1}=0$. In the appendix we show that this property also holds without the restrictions (3.3) to (3.5). This observation already characterizes an important difference between optimal emissions in case of substitutes and complements:

(1) If $S_{2}$ lies above the steady state, then $E_{1}$ should always be chosen higher for substitutes than it would be chosen if those pollutants were complements, regardless of $S_{1}$ :

$$
S_{2}(t)>S_{2}^{*} \rightarrow E_{1}\left(S_{1}, S_{2}, \omega=1\right)>E_{1}\left(S_{1}, S_{2}, \omega=-1\right)
$$

\footnotetext{
${ }^{5}$ The stead state shifts with $\omega$, but in this representation (deviation from steady state) the steady states for substitutes and complements coincide.
} 
(2) If $S_{2}$ lies below the steady state, then $E_{1}$ should always be chosen lower for substitutes than it would be chosen if those pollutants were complements, regardless of $S_{1}$ :

$$
S_{2}(t)<S_{2}^{*} \rightarrow E_{1}\left(S_{1}, S_{2}, \omega=1\right)<E_{1}\left(S_{1}, S_{2}, \omega=-1\right)
$$

The corresponding rule also holds for $E_{2}$ and $S_{1}$. The larger the difference between the stocks and their steady state values, the larger the difference between optimal emissions if those are complements or substitutes, respectively, with respect to the abatement cost function.

To focus on the issue of complementarity and substitutability we will restrict the analytical studies to the case of symmetric abatement costs and decay rates. We will allow, however, for different harmfulness of the pollutants, by assuming (3.3) and (3.4) but not (3.5). Thus the values for $\alpha_{i}$ may be different. W.l.o.g. the second pollutant is more harmful than the first $\left(\alpha_{1}=1, \alpha_{2} \geq 1\right)$.

The steady state obviously depends on $\omega$. However, enhancing $\omega$ (which also increases the overall abatement cost) does not necessarily increase the optimal steady state levels of both pollutants:

Proposition 3.2. Let (3.3) and (3.4) hold and assume (w.l.o.g.) $\alpha_{2} \geq \alpha_{1}=1$. Then the following holds:

(1) If $\omega$ rises, both the optimal steady state emissions and the steady state pollution stock rise for the less harmful pollutant, i.e., $\frac{d S_{1}^{*}}{d \omega}>0$ and $\frac{d E_{1}^{*}}{d \omega}>0$.

(2) For the more harmful pollutant the corresponding effects are ambiguous, i.e., $\frac{d S_{2}^{*}}{d \omega}>0$ and $\frac{d E_{2}^{*}}{d \omega}>0$.

The proofs of all propositions can be found in the appendix. Referring to the terminology introduced in section 2 (overlap of a level-effect and a substitution-effect) proposition 3.2 can be interpreted as follows: For the less harmful pollutant the leveland substitution-effect go into the same direction. For the more harmful pollutant these effects are counteracting. Here, it is in general not possible to determine which effect is dominating. ${ }^{6}$ Furthermore, one can show that the optimal paths need not to approach the steady state monotonically:

Proposition 3.3. Under assumptions (3.3) and (3.4) there exist initial stocks of pollution such that optimal emission paths do not behave monotonically.

\footnotetext{
${ }^{6}$ In fact, for most parameter values optimal stationary emissions will rise with $\omega$. However, they might fall, if the pollutants are very close substitutes $(\omega \approx \eta)$ and if the pollutant is comparatively more harmful than the other one (i.e. $\alpha_{2}>>\alpha_{1}$ ).
} 
This result contrasts with earlier findings on additively separable abatement costs and interaction of the pollutants in the damage function, where in case of identical decay rates all optimal emission paths behave monotonically over time (Moslener and Requate (2001)). Further analysis of both the eigenvalues and the eigenvectors leads to the following result:

Proposition 3.4. Let (3.3) and (3.4) hold. Further, let $\lambda_{\mathrm{s}}=\min \left[\left|\lambda_{a}\right|,\left|\lambda_{b}\right|\right]$ denote the "slow" eigenvalue. Then for the corresponding eigenvector $\left(\vec{v}_{\mathrm{s}}=\left(E_{1}^{\mathrm{s}}, E_{2}^{\mathrm{s}}, S_{1}^{\mathrm{s}}, S_{2}^{\mathrm{s}}\right)\right)$, satisfying equation (3.2) we obtain the following property concerning its components:

(1) For $\omega>0, \operatorname{sign}\left(E_{1}^{\mathrm{s}}\right)=\operatorname{sign}\left(E_{2}^{\mathrm{s}}\right)$, i.e., for substitutes either both emissions converge from above or both emissions converge from below towards the steady state.

(2) For $\omega<0, \operatorname{sign}\left(E_{1}^{\mathrm{s}}\right) \neq \operatorname{sign}\left(E_{2}^{\mathrm{s}}\right)$, i.e., for complements optimal emissions of one pollutant converge from above, while optimal emissions of the other pollutant converge from below towards the steady state.

The idea of the proof of propositions 3.3 and 3.4, given in the appendix, is to show that in the representation of the optimal paths, given by equation 3.2 , the $E_{1^{-}}$and $E_{2^{-}}$ components of the "fast" eigenvector have opposite signs for substitutes and identical signs for complements. By contrast, the eigenvectors of the "slow" eigenvector have identical (opposite) signs.

Put differently, for substitutes where an increase of $E_{1}$ along the iso-cost line tends to lower $E_{2}$ the allocation of emissions between $E_{1}$ and $E_{2}$ (say, $\frac{E_{2}}{E_{1}}$ ) is comparatively more important and happens on a faster time scale (motion along an eigenvector where the $E_{1}$ and $E_{2}$ components have opposite signs) than the aggregate pollution. The aggregate level of emissions (along the eigenvector where the $E_{1}$ and $E_{2}$-components have identical signs) is comparatively less important and converges towards the steady state on a slower time scale. Since in the neighborhood of the steady state the motion of the slow time scale always dominates, $E_{1}$ and $E_{2}$ will eventually converge towards the steady state either both from below or both from above. (In figure 1 (substitutes) both converge from below.)

For complements, by contrast, where along the iso-cost line a decrease of $E_{1}$ tends to lower $E_{2}$ as well, we would intuitively expect $E_{1}$ and $E_{2}$ to behave very similar. This means that the aggregate level of emissions is more important and should be adjusted on a faster time scale than the allocation between $E_{1}$ and $E_{2}$. As a consequence, the emissions of one pollutant will eventually converge towards the steady state from below, while the other will come from above. (See, e.g., figure 2.) The following result tells us how $\omega$ influences this motion: 
Proposition 3.5. Let assumptions (3.3) and (3.4) hold. Let $\lambda_{\mathrm{f}}=\max \left[\left|\lambda_{a}\right|,\left|\lambda_{b}\right|\right]$ denote the "fast" eigenvalue of (3.2). Then $\frac{\partial \lambda_{\mathrm{f}}}{\partial|\omega|}>0$. Therefore, a rising absolute value of $\omega$ induces the different optimal emissions to move faster in opposite (identical) directions if the pollutants are substitutes (complements).

A rising absolute value of $\omega$ means that the substitutability (or complementarity) is more pronounced. This implies a stronger (positive or negative) correlation between the two pollutants. Consequently, this increases the difference in the speed of the convergence processes described after proposition 3.4.

3.3. Remarks on the general case. Finally, some heuristic remarks on the general case without the symmetry restrictions (3.3) to (3.5) are in order: A higher harmfulness of pollutant $i$ (larger $\alpha_{i}$ ) leads to optimal emissions rules for $E_{1}$ and $E_{2}$ which are both steeper in the $S_{i}$-direction. (The planes in figure (5) would become steeper into the $S_{i}$-direction.) This is what one would expect since the stock $S_{i}$ of a more harmful pollutant should be more important for the optimal emissions. The angle between the optimal-emission-planes of pollutant $j$ for complements $(\omega=-1)$ and substitutes $(\omega=1)$ is increased if the harmfulness of the other pollutant $i$ rises (i.e., through an increase of $\alpha_{i}$ ). That means a rising $\alpha_{i}$ inflates the difference between the optimal

policy for pollutants being complements or substitutes. An increased ratio $\frac{\beta_{i}}{\beta_{j}}$ will have a similar effect on the policy rule. It means that pollutant $i$ decays comparatively slower than pollutant $j$, therefore being more harmful for the environment. This will again cause an increased difference between $\omega=1$ and $\omega=-1$ for the optimal emission level $E_{j}$.

We also refrain from pursuing a detailed analysis of the impact of the cost parameters. Intuitively one would expect that if abatement of, say pollutant 1 is comparatively costly (i.e. a higher $\eta_{1}$, other things equal), the optimal level of $E_{1}$ will vary less with $S_{1}$. In other words, for a pollutant with more costly abatement the influence of the pollutant stock is less strong (other things equal). This again feeds back into the optimal emissions rule for $E_{2}$ by making $S_{1}$ less important and therefore declining the difference between the optimal value of $E_{2}$ for complements and substitutes, respectively.

\section{IMPLEMENTATION OF THE OPTIMAL POLICY}

The optimal path, which can be non-monotonic, as formally described by (3.2) and as exemplified in figures 1 through 4 looks comparatively complex. Even if (as in our model) abatement cost and damage functions are perfectly known and both the steady state and the optimal emission path could be calculated ex ante, it is not obvious how such a path could be implemented. 
As we have noted above, for each pair of initial stocks of the two pollutants, there is an optimal path, as displayed in figures 3 and 4 . All these optimal paths are located in a 4-dimensional hyperplane. For each single pollutant $i=1,2$ we can draw a hyperplane in the $S_{1} / S_{2} / E_{i}$-space as we have done in figures 3 and 4 . These two hyperplanes can also be considered as functions $F_{i}: S_{1} \times S_{2} \rightarrow E_{i}$ which assign the optimal emission level (flow) of pollutant $i$ to each pair of pollution stocks. Note that once we are on that plane, the Ramsey conditions keep the emission path on the plane whereas the equations of motion for the pollution stocks (2.1) let the optimal path move along the plane.

Thus it is sufficient for the regulator to have at hand a pair of tables which represent the two hyperplanes $F_{i}$ and which exactly assign the optimal emission levels to each pair of pollution stocks observed by the regulator. The regulator can then issue two quantities of permits which exactly correspond to those optimal emission levels. The permits can only be used in the same period, i.e. banking and borrowing should not be allowed in this framework of perfect information. ${ }^{7}$ If the permit markets are competitive, the permit price will exactly reveal the optimal shadow price of pollution. If the regulator prefers a system of emission taxes instead of tradeable permits, similar tables can be set up for the optimal tax rates. In this way the regulator forces the emissions on the optimal path while the specific shape is determined by the dynamic nature of the pollutants.

Obviously establishing such tables in practice will not be as easy if the functional forms are not second order polynomials. However, since all differentiable functions can be approximated by a second order Taylor series around the steady state, the curvature properties are the same and the results remain valid. If one wants to deal with nonlinear costs and damages explicitly (or with stock-dependent decay rates) it is possible to generalize the notion of the policy plane to the notion of a policy 2-manifold. In this case it would not be possible to represent the optimal paths in terms of the eigenvectors but explicit simulations would have to be performed and the hyperplane would turn into a bent 2-dimensional manifold. However, in the tables this would merely change the numbers but not the mechanism.

The problem becomes more difficult, of either the cost or the damage function are explicitly time dependent. This would generate a non-autonomous system of coupled differential equations which cannot be represented in the phase space, meaning that the policy planes would no more exist in such a simple form.

\footnotetext{
${ }^{7}$ Banking and borrowing could be useful in a framework of aggregate uncertainty. See Yates and Cronshaw (2001), or Phaneuf and Requate (2001).
} 


\section{Conclusions And FURTher RESEARCH}

We have set up a model for several accumulating pollutants where abatement of different pollutants (causing different externalities) does not happen independently. For illustrative purposes we have focused on the case of two pollutants for the most part of the paper. For a given (abatement) technology the pollutants can be either complements or substitutes. Examples for complements may inter alia be efficiency improvements, examples for substitutes may be end-of-pipe technologies which cause other emissions, such as $\mathrm{CO}_{2}$ from energy use.

For pollutants with symmetric abatement costs and identical decay rates optimal steady state emissions turned out to rise with the degree of substitutability for the less harmful pollutant while this effect was ambiguous for the more harmful one. Moreover, optimal paths for complements and substitutes show different behavior. This remains valid even when the pollutants and other model variables are highly symmetric.

In general our analysis has shown that environmental policy based on the isolated analysis of only one pollutant may be qualitatively misleading if other pollutants are involved.

\section{Appendix: Proofs of the Propositions}

Policy planes of complements and substitutes are mirror images. We will now show that the policy planes of complements and substitutes through the steady state are mirror images of each other with respect to the corresponding $S_{i}$-plane. The eigenvalue problem from equations (2.1) and (2.6) which yields the eigenvalues and eigenvectors spanning the policy planes reads

$$
\Sigma(\omega) \cdot\left(\begin{array}{c}
E_{1} \\
E_{2} \\
S_{1} \\
S_{2}
\end{array}\right)=\left(\begin{array}{c}
\lambda E_{1} \\
\lambda E_{2} \\
\lambda S_{1} \\
\lambda S_{2}
\end{array}\right)
$$

with the Jacobian $\Sigma(\omega)$ depending on $\omega$ :

$$
\begin{gathered}
\Sigma(\omega)= \\
\left(\begin{array}{cccc}
{\left[\left(\beta_{1}+r\right) \eta_{1} \eta_{2}-\left(\beta_{2}+r\right) \omega^{2}\right]} & \omega \eta_{2}\left(\left(\beta_{1}+r\right)-\left(\beta_{2}+r\right)\right) & \eta_{2} & -\alpha_{2}^{2} \omega \\
\omega \eta_{1}\left(\left(\beta_{2}+r\right)-\left(\beta_{1}+r\right)\right) & {\left[\left(\beta_{2}+r\right) \eta_{1} \eta_{2}-\left(\beta_{1}+r\right) \omega^{2}\right]} & -\omega & \alpha_{2}^{2} \eta_{1} \\
1 & 0 & -\beta_{1} & 0 \\
0 & 1 & 0 & -\beta_{2}
\end{array}\right) .
\end{gathered}
$$


To establish that the eigenvectors are transformed into their mirror images when moving from $\omega$ to $-\omega$ we need to show: $\left(E_{1}, E_{2}, S_{1}, S_{2}\right)$ solves the eigenvalue equation for $\omega$ if $\left(E_{1},-E_{2}, S_{1},-S_{2}\right)$ solves it for $-\omega$ lst $^{8}$.

$$
\Sigma(\omega) \cdot\left(\begin{array}{c}
E_{1} \\
E_{2} \\
S_{1} \\
S_{2}
\end{array}\right)=\left(\begin{array}{c}
\lambda E_{1} \\
\lambda E_{2} \\
\lambda S_{1} \\
\lambda S_{2}
\end{array}\right) \Leftrightarrow \Sigma(-\omega) \cdot\left(\begin{array}{c}
E_{1} \\
-E_{2} \\
S_{1} \\
-S_{2}
\end{array}\right)=\left(\begin{array}{c}
\lambda E_{1} \\
-\lambda E_{2} \\
\lambda S_{1} \\
-\lambda S_{2}
\end{array}\right)
$$

We now investigate what will happen to the Jacobian if $\omega$ changes sign: The parameter $\omega$ only occurs in the two upper rows. If it occurs only quadratic, its sign does not matter. Therefore, the sign only changes (i) in row 1 (column 2 and 4); (ii) in row 2 (columns 1 and 3). The equivalence (5.3) can be confirmed by simple calculation.

Proposition 3.2. Setting $E_{i}^{*}=S_{i}^{*}=0$ the steady-state values turn out to be:

$$
S_{i}^{*}=\frac{1}{d e t} \cdot e^{\max }(r+\beta)\left[\alpha_{i}^{2}+\beta(r+\beta)(\eta-\omega)\right](\eta-\omega)
$$

where

$$
\operatorname{det}=\alpha_{2}^{2}+\eta \beta\left(1+\alpha_{2}^{2}\right)(r+\beta)+\beta^{2}(r+\beta)^{2}\left(\eta^{2}+\omega^{2}\right) .
$$

By assumption $a_{2} \geq a_{1}=1$ we show $\frac{\partial S_{1}^{*}}{\partial \omega}>0$ and $\frac{\partial S_{2}^{*}}{\partial \omega}$ to be ambiguous in sign.

$$
\begin{aligned}
\frac{d S_{1}^{*}}{d \omega} & =\frac{1}{d e t^{2}} \cdot e^{\max }(r+\beta) \\
& \cdot\left[\alpha_{2}^{4}+\alpha_{2}^{2} \beta(r+\beta)\left(\eta+\alpha_{2}^{2} \eta-2 \omega\right)+\beta^{2}(r+\beta)^{2}\left(\alpha_{2}^{2}\left(\eta^{2}+\omega^{2}\right)-2 \eta \omega\right)\right]
\end{aligned}
$$

The denominator is clearly positive. Recalling $|\eta|>|\omega|$ we see that the numerator is positive as well, since $\eta+\alpha_{2}^{2} \eta-2 \omega \geq 2 \eta-2 \omega>0$, and $\alpha_{2}^{2}\left(\eta^{2}+\omega^{2}\right)-2 \eta \omega>$ $\eta^{2}-2 \eta \omega+\omega^{2}=(\eta-\omega)^{2}>0$.

$$
\begin{aligned}
\frac{d S_{2}^{*}}{d \omega} & =\frac{1}{d e t^{2}} \cdot e^{\max }(r+\beta) \\
& \cdot\left[\alpha_{2}^{2}+\beta(r+\beta)\left(\eta+\alpha_{2}^{2} \eta-2 \alpha_{2}^{2} \omega\right)+\beta^{2}(r+\beta)^{2}\left(\eta^{2}+\omega^{2}-2 \alpha_{2}^{2} \eta \omega\right)\right]
\end{aligned}
$$

It is easy to confirm that $\frac{\partial S_{2}^{*}}{\partial \omega}$ may be positive or negative: Setting $\omega=0$, we see that the enumerator is positive. Setting $\omega \approx \eta$, we see that $\alpha_{2}$ can be chosen large enough to lead to a negative enumerator.

The latter proves the proposition and shows that for good substitutes we tend to have falling stationary state emissions of the more harmful pollutant when $\omega$ rises.

\footnotetext{
${ }^{8}$ Eigenvectors are invariant with respect to their sign and multiplying by a scalar. It is therefore equivalent to have the minus-sign at $E_{2}$ and $S_{2}$ or at $E_{1}$ and $S_{1}$.
} 
Proposition 3.3. Recall that the optimal path into the steady state can be described by motion along different directions (eigenvectors) with different characteristic speeds (eigenvalues). It is therefore sufficient to show that there exists no eigenvector which has vanishing components in the $E_{i}$-directions, i.e. $E_{1}=E_{2}=0$. This can be done indirectly by making use of the eigenvalue-equations. (In principle it is also necessary to show that the eigenvalues are different, but this can directly be seen from their expressions which are explicitly displayed in the proof of proposition 3.) The eigenvalue problem is defined by

$$
\Sigma \cdot\left(\begin{array}{c}
E_{1} \\
E_{2} \\
S_{1} \\
S_{2}
\end{array}\right)=\left(\begin{array}{c}
\lambda E_{1} \\
\lambda E_{2} \\
\lambda S_{1} \\
\lambda S={ }_{2}
\end{array}\right)
$$

where

$$
\boldsymbol{\Sigma}=\left(\begin{array}{cccc}
{\left[\left(\beta_{1}+r\right) \eta_{1} \eta_{2}-\left(\beta_{2}+r\right) \omega^{2}\right]} & \omega \eta_{2}\left(\left(\beta_{1}+r\right)-\left(\beta_{2}+r\right)\right) & \eta_{2} & -\alpha_{2}^{2} \omega \\
\omega \eta_{1}\left(\left(\beta_{2}+r\right)-\left(\beta_{1}+r\right)\right) & {\left[\left(\beta_{2}+r\right) \eta_{1} \eta_{2}-\left(\beta_{1}+r\right) \omega^{2}\right]} & -\omega & \alpha_{2}^{2} \eta_{1} \\
1 & 0 & -\beta_{1} & 0 \\
0 & 1 & 0 & -\beta_{2}
\end{array}\right)
$$

Setting $E_{1}=E_{2}=0$ would yield

$$
\begin{aligned}
\lambda & =-\beta \\
\eta S_{1}-\alpha_{2}^{2} \omega S_{2} & =0 \\
-\omega S_{1}+\alpha_{2}^{2} \eta S_{2} & =0 .
\end{aligned}
$$

This implies $\omega=\eta$ which is in contradiction to the assumption of our cost function.

To construct a non-monotonic emission path one just has to choose the initial conditions in such a way that the optimal emission path first moves upwards along the eigenvector with the "fast" eigenvalue and downwards along the eigenvector with the "slow" eigenvalue.

Proposition 3.4. We will show that for complements $(\omega>0)$ the negative eigenvalue of an eigenvector which has positive $E_{1}$ and $E_{2}$-components has a smaller absolute value than the one that corresponds to the eigenvector with different signs of $E_{1}, E_{2}$.

All four eigenvalues of the problem (5.1) can be represented as follows:

$$
\lambda_{a, b, c, d}=\frac{1}{2}\left(r \pm \frac{\sqrt{\left(\eta^{2}-\omega^{2}\right)\left((r+2 \beta)^{2}\left(\eta^{2}-\omega^{2}\right)+2\left(\eta\left(1+\alpha^{2}\right) \pm \sqrt{\left(\alpha^{2}-1\right)^{2} \eta^{2}+4 \alpha^{2} \omega^{2}}\right)\right)}}{\eta^{2}-\omega^{2}}\right) .
$$


The eigenvectors of the stable branches are the negative ones, meaning that the first $( \pm)$ (before the outer squareroot) has to be $(-)$ for the stable branches. Instead of the indices $a$ and $b$ we will use $s$ and $f$ to indicate the eigenvalues belonging to the slow and fast motion.

The negative eigenvalues for the symmetric case are therefore:

$$
\lambda_{f, s}=\frac{1}{2}\left(r-\frac{\sqrt{\left(\eta^{2}-\omega^{2}\right)\left((r+2 \beta)^{2}\left(\eta^{2}-\omega^{2}\right)+2\left(\eta\left(1+\alpha^{2}\right) \pm \sqrt{\left(\alpha^{2}-1\right)^{2} \eta^{2}+4 \alpha^{2} \omega^{2}}\right)\right)}}{\eta^{2}-\omega^{2}}\right) .
$$

with $(+)$ for the fast and $(-)$ for the slow motion. The corresponding eigenvectors have the components

$$
\begin{gathered}
E_{1}=1 \\
E_{2 f, s}=\frac{\eta\left(1-\alpha^{2}\right) \mp \sqrt{\eta^{2}\left(\alpha^{2}-1\right)^{2}+4 \alpha^{2} \omega^{2}}}{2 \alpha^{2} \omega}
\end{gathered}
$$

where we have chosen $E_{1}$ and calculated $E_{2}$ accordingly ${ }^{9}$. Here $(-)$ represents the fast and $(+)$ the slow motion. For $\omega>0$ it follows that $E_{2}<0$ for the fast motion and $E_{2}>0$ for the slow motion. For $\omega<0$ the signs are reversed. This proves the proposition.

Proposition 3.5. Analyzing the comparative dynamics of $\left|\lambda_{f}\right|$ we obtain:

$$
\frac{d \lambda_{f}}{d \omega}=\frac{1}{B(>0)} \cdot(-\omega)\left[\left(1+\alpha^{4}\right) \eta^{2}+2 \alpha^{2} \omega^{2}+\left(1+\alpha^{2}\right) \eta \sqrt{\left(\alpha^{2}-1\right)^{2} \eta^{2}+4 \alpha^{2} \omega^{2}}\right] .
$$

Where $B$ is a lengthy but clearly positive expression. It is therefore irrelevant for the sign of $\frac{d \lambda_{f}}{d \omega}$ and we just show it for the sake of completeness:

$$
\begin{aligned}
B= & \left(\eta^{2}-\omega^{2}\right) \sqrt{\left(\alpha^{2}-1\right)^{2} \eta^{2}+4 \alpha^{2} \omega^{2}} \times \\
& \sqrt{\left(\eta^{2}-\omega^{2}\right)\left((r+2 \beta)^{2}\left(\eta^{2}-\omega^{2}\right)+2\left(\eta\left(1+\alpha^{2}\right)+\sqrt{\left(\alpha^{2}-1\right)^{2} \eta^{2}+4 \alpha^{2} \omega^{2}}\right)\right)} .
\end{aligned}
$$

For $\omega>0$ we see $\frac{d \lambda_{f}}{d \omega}<0$, and for $\omega<0$ we can confirm $\frac{d \lambda_{f}}{d \omega}>0$. Thus in both cases an increasing $|\omega|$ increases $\left|\lambda_{f}\right|$.

\section{REFERENCES}

Böhringer, C., A. Löschel, and T. F. Rutherford: (forthcoming), "Efficiency Gains from 'What'-Flexibility in Climate Policy - An Integrated CGE Assessment", The Energy Journal.

\footnotetext{
${ }^{9}$ This is always possible by renormalization of the eigenvector.
} 
Burniaux, J.-M.: (2000), "A Multi-Gas Assessment of the Kyoto Protocol", OECD Economics Department Working Paper No. 270.

Cansier, A. and D. Cansier: (1999), "Umweltstandards bei Unsicherheit aus Entscheidungstheoretischer Sicht", Tübinger Diskussionsbeitrag, No. 170.

Chiang, A.C.: (1992), "Elements of Dynamic Optimization", McGraw-Hill, Singapore.

Endres, A.: (1985), "Environmental Policy with Pollutant interaction", in Rüdiger Pethig, ed., Public Goods and Public Allocation Policy, Frankfurt/M.

Feichtinger, G. and R.F. Hartl: (1986), "Optimale Kontrolle ökonomischer Prozesse", Walter de Gruyter, Berlin.

Leightner, J.E.: (1999), "Weather-induced changes in the tradeoff between $\mathrm{SO}_{2}$ and $\mathrm{NO}_{x}$ at large power plants", Energy Economics 21, 239-59.

Manne, A. and R. Richels: (2000), "A Multi-Gas Approach to Climate Policy - with and without GWPs", Paper presented at the EMF-19 Workshop in Washington DC.

Michaelis, P.: (1996), "Effiziente Klimapolitik im Mehrschadstoffall", J.C.B. Mohr, Tübingen.

Michaelis, P.: (1999), "Sustainable Greenhouse Policies: The Role of Non- $\mathrm{CO}_{2}$ Gases", Structural Change and Economic Dynamics 10, 239-260.

Moslener, U. and T. Requate: (2001), "Optimal Abatatement Strategies for Various Interacting Greenhouse Gases", Discussion Paper Series, University of Heidelberg 360.

Nordhaus, W.D.: (2000), "Warming the World, Economic Models of Global Warming", MIT Press, Cambridge.

Phaneuf, D. and T. Requate: (2002), "Incentives for Investment in Advanced Pollution Abatement Technology in Emission Permit Markets with Banking", Environmental and Resource Economics 22 (3), 369-390.

Reilly, J. et al.: (1999), "Multi-gas assessment of the Kyoto Protocol", Nature 401, 549-555.

Repetto, R.: (1987), "The Policy Implications of Non-convex Environmental Damages: A Smog Control Case Study", Journal of Environmental Economics and Management 14, 13-29.

Yates, A. and M. Cronshaw: (2001), "Pollution Permit Markets with Intertemporal Trading and Asymmetric Information", Journal of Environmental Economics and Management 42 (1), 104-118. 